\title{
Modelado Numérico del Socavado Lateral en Procesos de Soldadura
}

\author{
A. Cassanelli, A. Márquez $z^{\mathrm{a}}$, G. Lombera ${ }^{\mathrm{a}}$, L.A. De Vedia ${ }^{\mathrm{a}, \mathrm{b}}$ \\ ${ }^{a}$ Grupo De Ingeniería Asistida Por Computadora, \\ Dpto. De Mecánica,Facultad De Ingeniería, Unmdp, Mar Del Plata, Argentina \\ ${ }^{b}$ Instituto De Tecnología Prof. Jorge A. Sábato (UNSAM-CNEA), \\ CIC, Buenos Aires, Argentina
}

Received: May 6, 2002; Revised: December 5, 2002

\begin{abstract}
When welded joints have to meet particular requirements related to fatigue life they are usually designed using the so called S-N design curves. This implies the construction of a curve for each particular design. From the point of view of the user, it is often difficult to establish accurately which is the curve that better fits the design that is being considered. The so called "local approach " is based on the concept that the fatigue strenght of welded joints is essentially determined by the local stress and strain in the " hot spots ". These spots are located in the geometric discontinuities of the joint. Through this approach is possible to consider the possibility of constructing and using only one curve of design or " key curve ", for a given material. In this case the design parameter is the local stress or strain instead of the nominal stress. In the present work the lateral defect is modeled with fillet welds using the local approach combined with the finite elements method. A data base of Kt for different dimensionless geometric relations is generated, and from these results an analytical function for the value of the concentrator is interpolated. Finally the contour plots that were generated help reducing the number laboratory tests needed for the determination of Kt.
\end{abstract}

Keywords: mechanic design, fatigue, local approach

\section{Introducción}

En presencia de cargas fluctuantes, en el vértice de discontinuidades geométricas mas o menos agudas se produce un fenómeno de deformación elasto-plástica cíclica a partir de la cual se produce la iniciación de la fisura por fatiga.

La naturaleza esencialmente multiparamétrica del fenómeno de fatiga, en el que la influencia de los distintos parámetros no puede en general considerarse de manera aislada, constituye la razón de la gran dispersión que generalmente acompaña a los resultados experimentales relacionados con este fenómeno.

La influencia que las discontinuidades geométricas tienen sobre la resistencia a la fatiga de las uniones soldadas sobre su comportamiento en servicio bajo régimen de cargas variables es un hecho ampliamente reconocido ${ }^{1-5}$. En tal sentido, el talón de la soldadura, sea esta a tope o a filete, es el asiento de las discontinuidades que en general son los factores limitantes de la vida a la fatiga de uniones soldadas solicitadas transversalmente al cordón de soldadura $^{1-6}$.

La manera habitual de encarar el diseño de uniones soldadas con una vida a la fatiga compatible con los requerimientos particulares del diseño que se trate, es a través del empleo de las denominadas "curvas de diseño" S-N, (donde $\mathrm{S}$ es la amplitud de tensiones resultante de las cargas externas aplicadas y $\mathrm{N}$ el número de ciclos a amplitud de tensiones constante que conduce a la falla) incorporadas a los códigos de construcción o a otros documentos aplicables $^{1-3}$.

Tal metodología posee por un lado el inconveniente que es necesario construir una curva de diseño particular para cada diseño de detalle de unión soldada, y desde el punto de vista del usuario de las curvas, resulta muchas veces dificultoso establecer con precisión cuál de aquellas es la que mejor se corresponde con el diseño de detalle

*e-mail: glombera@fi.mdp.edu.ar 
particular que se esté considerando.

Una forma racional de encarar el problema de diseño a la fatiga de uniones soldadas puede sustentarse en la consideración del hecho que para cualquier unión soldada sujeta a cargas cíclicas, los puntos críticos a partir de los cuales las fisuras por fatiga son generadas, están usualmente localizados en el talón de la soldadura o en la raíz, razón por la cual estos puntos han sido bautizados comunmente como "puntos calientes"("hot spots"). El denominado "enfoque local" se basa en el concepto que la resistencia a la fatiga de una unión soldada está esencialmente determinada por las tensiones y deformaciones actuantes localmente en el "punto caliente", es decir en la discontinuidad geométrica que constituye el concentrador de tensiones más eficaz que contenga la unión (para la dirección de las cargas actuantes)

De acuerdo con esta idea, la tensión total que actúa en el "punto caliente", es la resultante de la composición de los siguientes efectos:

a. La tensión nominal $\mathrm{s}_{\mathrm{N}}$ que resulta de la aplicación de las cargas externas, calculada con las fórmulas usuales de la teoría ingenieril de vigas o de resistencia de materiales.

b. La tensión estructural $\mathrm{s}_{\mathrm{S}}=\mathrm{K}_{\mathrm{S}} \mathrm{s}_{\mathrm{N}}$, que resulta de tener en cuenta la geometría estructural de la unión, donde $\mathrm{K}_{\mathrm{S}}$ es el factor de amplificación de tensiones por la forma global de la unión. Esta tensión afecta a una porción importante de la sección e influye por lo tanto no sólo en la iniciación sino también en la propagación de la fisura por fatiga.

c. La tensión pico local $\mathrm{s}_{\mathrm{L}}=\mathrm{K}_{\mathrm{t}} \mathrm{s}_{\mathrm{S}}=\mathrm{K}_{\mathrm{t}} \mathrm{K}_{\mathrm{S}} \mathrm{s}_{\mathrm{N}}$ donde $\mathrm{K}_{\mathrm{t}}$, es el factor teórico elástico de concentración de tensiones introducido por la presencia del concentrador local constituido por el defecto de socavado y la geometría local del perfil del cordón de soldadura. Esta es una tensión local que sólo tiene influencia en la etapa de iniciación, ya que se extingue rápidamente al progresar la fisura.

d. La geometría de la unión es también perturbada por la presencia de otros defectos inducidos por el proceso de soldadura, de modo que se podría introducir una nueva tensión $\mathrm{s}_{\mathrm{W}}=\mathrm{K}_{\mathrm{w}} \mathrm{s}_{\mathrm{L}}=\mathrm{K}_{\mathrm{w}} \mathrm{K}_{\mathrm{t}} \mathrm{s}_{\mathrm{S}}$, donde $\mathrm{K}_{\mathrm{w}}$ es el factor de concentración de tensiones producido por una distribución estadística de defectos en la soldadura.

El problema consiste entonces en establecer cuál de las tensiones mencionadas define el daño por fatiga de modo de utilizarla como tensión de diseño. Algunos autores ${ }^{1}$ sugieren que la consideración de la tensión estructural $\mathrm{s}_{\mathrm{S}}$ es adecuada porque asumen que el factor de concentración local $\mathrm{K}_{\mathrm{t}}$ se mantiene esencialmente constante para un proceso en particular. Este concepto no es del todo correcto y un mismo proceso puede generar por un cambio en los parámetros de soldadura, modificaciones importantes en la geometría local del socavado con la consiguiente variación en los valores de $\mathrm{K}_{\mathrm{t}}$.

En cambio, la distribución estadística de los otros defectos puede considerarse como esencialmente invariable para una dada industria trabajando con un dado estándar de calidad, y por lo tanto el factor $\mathrm{K}_{\mathrm{w}}$ puede asumirse razonablemente como constante para cada aplicación en particular. La variable mas apropiada para caracterizar la vida a la fatiga de la unión soldada hasta la iniciación o nucleación de la fisura es la tensión pico local $\mathrm{s}_{\mathrm{L}}$, mientras que la propagación subcrítica estará básicamente controlada por $\mathrm{s}_{\mathrm{S}}^{2,3}$.

Ahora bien, un problema que se presenta es que muchas veces no existen expresiones de cálculo desarrolladas para obtener el valor de $\mathrm{K}_{\mathrm{t}}$, considerándose que este valor está implícito en las curvas de diseño. Otro problema es el constituido por el hecho que debido al efecto de micro soporte elasto-plástico que tiene lugar en el vértice de la discontinuidad, $\mathrm{K}_{\mathrm{t}}$ no describe adecuadamente la reducción en la vida a la fatiga de la soldadura en relación con la que corresponde a un espécimen sin entalla del mismo material. Esta reducción es tenida en cuenta utilizando un factor de entalla a la fatiga $\mathrm{K}_{\mathrm{f}}$ en términos de $\mathrm{K}_{\mathrm{t}} \mathrm{y}$ del conocimiento del comportamiento cíclico del material. De este modo se puede considerar la posibilidad de construir y utilizar una curva de diseño única o "key curve", para un dado material en el que el parámetro de diseño sea la tensión o la deformación local en lugar de la tensión nominal como ocurre en la actualidad. La idea central que sustenta este enfoque es que el comportamiento a la fatiga del material adyacente al vértice del concentrador, está adecuadamente representado por el comportamiento de una probeta sin entalla sometida a las mismas tensiones y deformaciones que experimenta el material adyacente al vértice del concentrador.

En el presente trabajo se realiza un estudio paramétrico de las variables geométricas de la discontinuidad, con el objeto de encontrar una expresión analítica del parámetro $\mathrm{K}_{\mathrm{t}}$.

\section{Geometría Adoptada}

La geometría del cordón generado por el proceso de soldadura posee un perfil irregular que se puede aproximar mediante una geometría simplificada como se puede ver en Fig.1. La altura del cordón de soldadura, el espesor de chapa y el radio del socavado y el ángulo de los laterales del mismos son las variables geométricas consideradas.

En el presente trabajo se consideró el ancho del cordón de soldadura igual al espesor de chapa y el ángulo de los laterales del socavado se fijó constante de valor $60^{\circ}$.

En la Tabla 1, se muestran los rangos de variación de los parámetros geométricos. 


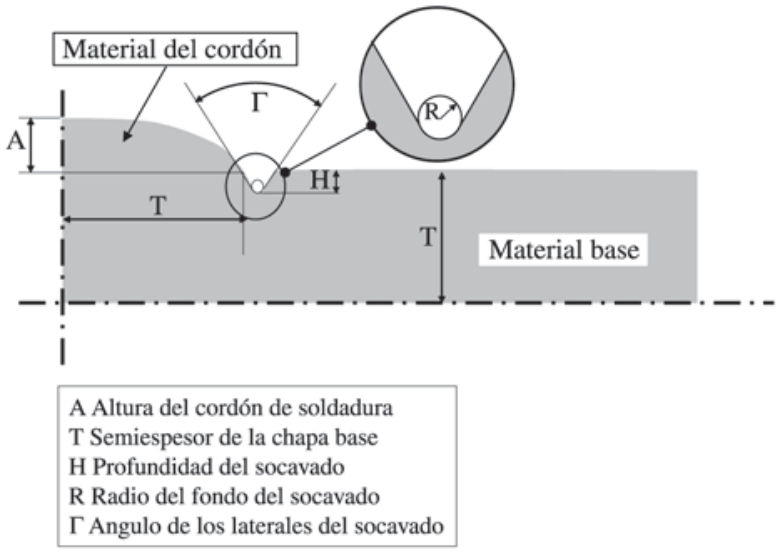

Figura 1. Geometria simplificada del cordón de soldadura.

Tabla 1. Rangos de variación de los parámetros geométricos.

\begin{tabular}{|c|c|c|c|}
\hline \multicolumn{4}{|c|}{ Rango de Variación de los Parámetros } \\
\hline Descripcion & Número & Rango & Variación \\
\hline ecifica & $\mathrm{A} / \mathrm{T}$ & $0.10-0.50$ & 0.1 \\
\hline $\begin{array}{l}\text { Profundidad especifica } \\
\text { del Socavado }\end{array}$ & $\mathrm{H} / \mathrm{T}$ & $0.50-0.15$ & 0.5 \\
\hline Agudeza del socavado & $\mathrm{R} / \mathrm{H}$ & $0.05-2.0$ & GEOM 2.5 \\
\hline Parámetro $\Gamma$ & $\Gamma$ & $60^{\circ}$ & Cte. \\
\hline
\end{tabular}

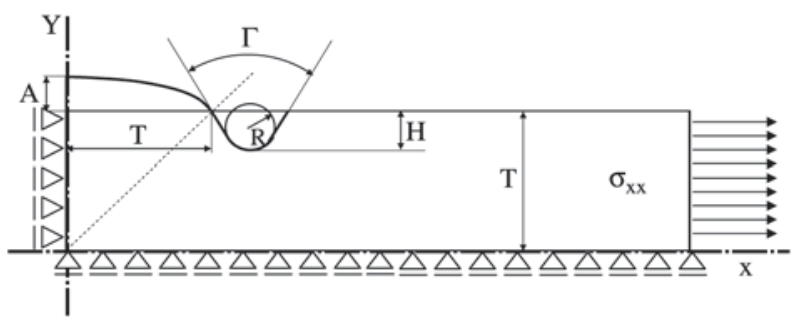

Figura 2. Condiciones de contorno

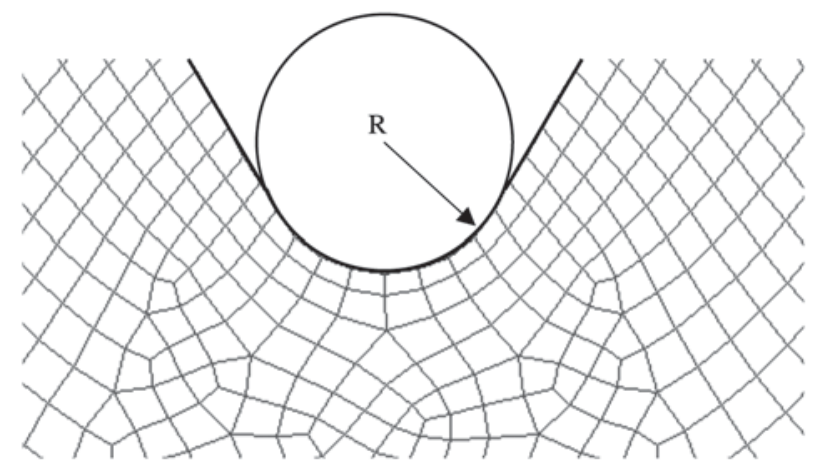

Figura 3. Malla de elementos finitos.

\section{Método de resolución}

Se consideró una sección de un perfil de soldadura a tope. Se establecieron condiciones de simetría horizontal y vertical por las secciones medias. Se aplicó una solicitación de tracción uniforme $\mathrm{s}_{x x}=1$, Fig. 2. Para la resolución se utilizó el método de los elementos finitos con un esquema elástico en deformación plana.

La puesta a punto del modelo se realizó con el caso definido por: $R / H$ : 2, H/T: 0.05, A/T: 0.1. Mediante un análisis recurrente de sensibilidad y convergencia de la

solución numérica se ajustaron los parámetros del generador de malla de elementos finitos. En la Fig. 3 se muestra el mallado en la zona del socavado adyacente al cordón de soldadura.

\section{Resultados y discusión}

En el presente trabajo se realizó un estudio sistematizado del factor $\mathrm{Kt}$, teniendo en cuenta que es independiente de la carga nominal aplicada y de las dimensiones absolutas del modelo en el rango elástico. Kt puede ser expresado en función de los parámetros geométricos adimensionales ${ }^{22}$ dados en la Tabla 1.

El valor máximo de tensión normal $s_{x x}$ encontrado se adopta como el valor del concentrador de tensión $K t$, definida la tensión remota $s_{N}$ de valor unitario.

Se obtuvo una base de datos de Kt a partir de 75 modelos generados con 5 valores de A/T y 5 valores de $\mathrm{R} / \mathrm{H}$ para $\mathrm{H} / \mathrm{T} 0.05,0.10$ y 0.15 respectivamente que pueden verse en la Tabla 2. Los valores de Kt resultantemuestran una corres-

Tabla 2. Kt en función de los parámetrosgeométricos adimendionales

\begin{tabular}{ccccccc}
\hline & & & & $\mathrm{A} / \mathrm{T}$ & & \\
$\mathrm{R} / \mathrm{H}$ & $\mathrm{H} / \mathrm{T}$ & 0.1 & 0.2 & 0.3 & 0.4 & 0.5 \\
\hline 0.05 & & 13.8 & 15.38 & 16.4 & 16.8 & 17.08 \\
0.13 & & 8.92 & 10 & 10.5 & 10.8 & 11 \\
0.30 & 0.05 & 6.07 & 6.76 & 7.14 & 7.36 & 7.43 \\
0.75 & & 4.19 & 4.6 & 4.8 & 4.91 & 4.95 \\
2.00 & & 2.88 & 3.02 & 3.17 & 3.259 & 3.331 \\
0.05 & & 12.6 & 13.4 & 13.9 & 14.1 & 14.2 \\
0.13 & & 8.196 & 8.785 & 9.037 & 9.216 & 9.279 \\
0.30 & 0.10 & 5.534 & 5.863 & 6.06 & 6.16 & 6.2 \\
0.75 & & 3.79 & 3.934 & 4.1 & 4.158 & 4.183 \\
2.00 & & 2.602 & 2.7 & 2.786 & 2.825 & 2.833 \\
0.05 & & 12 & 12.5 & 13 & 12.9 & 13 \\
0.13 & & 7.86 & 8.13 & 8.3 & 8.39 & 8.47 \\
0.30 & 0.15 & 5.24 & 5.51 & 5.64 & 5.69 & 5.72 \\
0.75 & & 3.652 & 3.746 & 3.818 & 3.848 & 3.861 \\
2.00 & & 2.511 & 2.585 & 2.606 & 2.626 & 2.632 \\
\hline
\end{tabular}




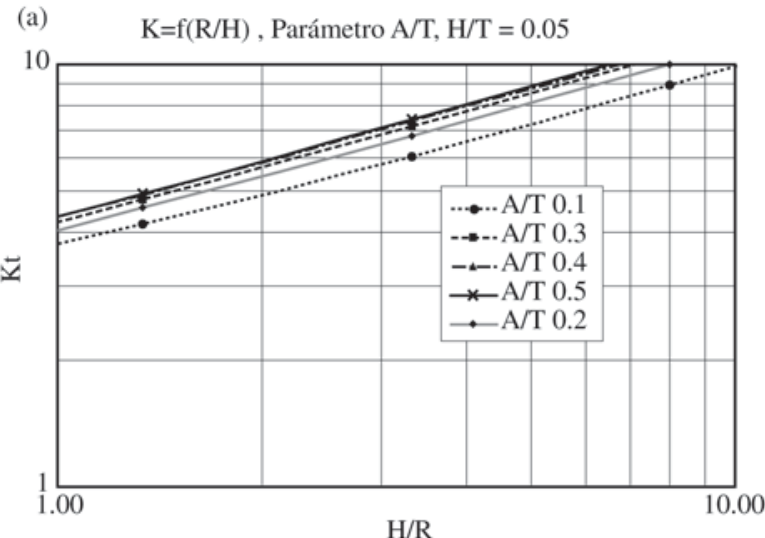

(b)

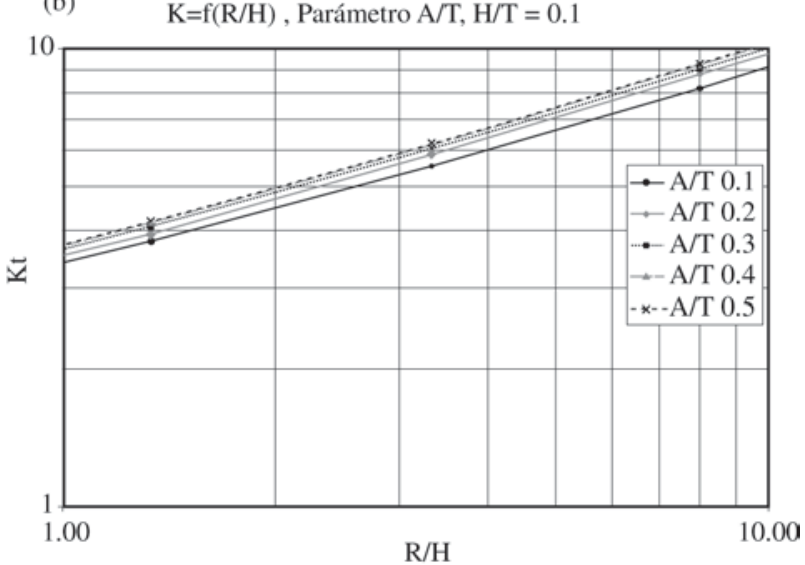

(c)

$\mathrm{K}=\mathrm{f}(\mathrm{R} / \mathrm{H})$, Parámetro $\mathrm{A} / \mathrm{T}, \mathrm{H} / \mathrm{T}=0.15$

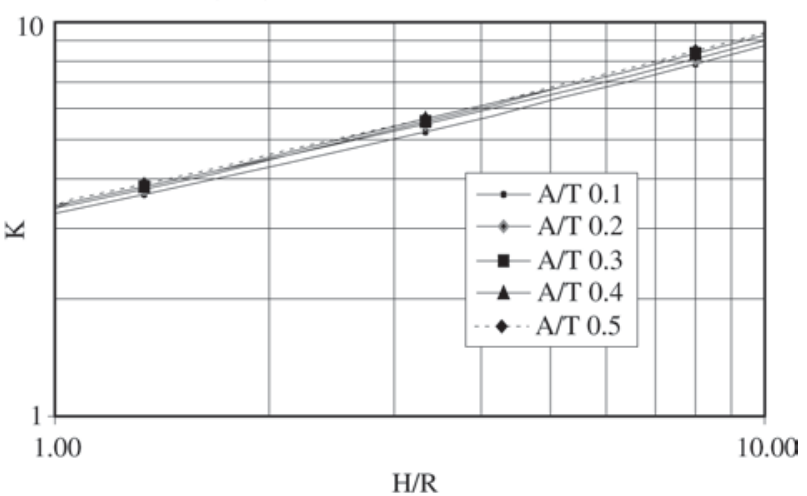

Figura 4. Valores de Kt en función de H/R para distintos valores de $\mathrm{H} / \mathrm{T}$.

pondencia con la variación de la severidad del defecto. Sin embargo, algunos valores obtenidos para algunas relaciones geométricas muestran una elevación de la tensión local poco sostenible estructuralmente en la práctica, como por ejemplo Kt 17.08 con (R/H 0.05, H/T 0.05 y A/T 0.5).

En la Fig. 4 (a, b y c) se representan valores de $K_{t}$ en

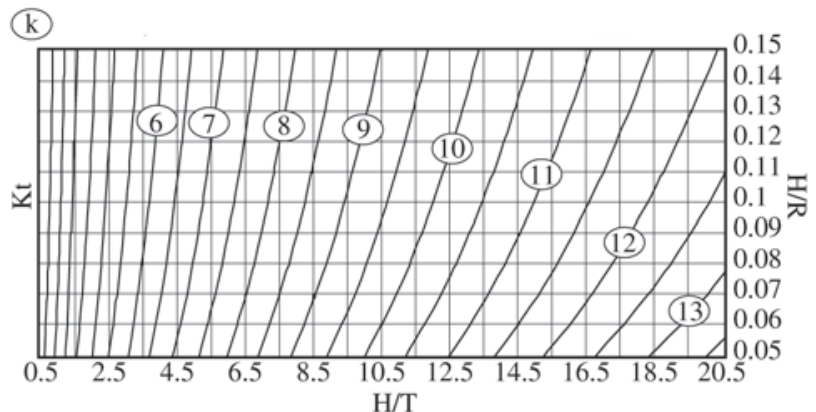

Figura 5. Kt en función de H/T y H/R para A/T = 0.05

función de $H / R$ en escala doble logarítmica para las relaciones $\mathrm{H} / \mathrm{T} 0.05,0.5$ y 0.15 respectivamente. Se observa un comportamiento lineal que permite encontrar una expresión analítica para el valor de Kt en función de las variables adimensionales dada por la siguiente ecuación:

$\mathrm{K}_{\mathrm{t}}=(-1.137 \mathrm{~A} / \mathrm{T}+2.6789) \times \mathrm{H} / \mathrm{T}^{(-0.3264 * \mathrm{~A} / \mathrm{T}-0.097)} \times \mathrm{H} / \mathrm{R}^{0.434}$

La diferencia máxima observada entre la función de Kt y los valores de la base de datos es en el peor de los casos menor al 5\%. Para extrapolaciones con valores extremos de $\mathrm{R} / \mathrm{H}(0.01$ y 10$)$, la diferencia fue menor al $10 \%$, alcanzándose un buen acuerdo con esta expresión.

Para una mejor planificación de ensayos de laboratorio para la determinación experimental de Kt, es necesario diferenciar los grupos de relaciones geométricas que generan el mismo valor del concentrador. En tal sentido se pueden generar curvas de nivel para Kt utilizando la ecuación encontrada que permiten identificar conjuntos de valores de los parámetros adimensionales que corresponden a un mismo valor de elevación local de tensiones. A modo de ejemplo se puede ver en la Fig. 5 curvas de nivel de Kt para relación A/T 0.05 .

\section{Conclusiones}

En este trabajo fue posible modelar mediante el uso del método de los elemento finitos una geometría simplificada del defecto de socavado lateral en una soldadura de chapa a tope. La selección y parametrización de las variables geometricas (semiespesor de la chapa "T", profundidad del defecto " $H$ ", el radio del socavado "R" y la altura del cordón "A") para adimensionalizar el modelo fue consistente con el problema real.

Con objeto de obtener la condición mas severa de restricción a la deformación en el área del defecto se adopto un esquema de deformacion plana y material elástico lineal.

Se optimizaron los recursos informáticos con un refinamiento apropiado de la maya de elementos finitos, ajustando los parámetros del generador mediante un proceso recu- 
rrente a partir de una solucion teorica dada de Kt.

Los valores de Kt resultantes muestran una correspondencia con la variación de la severidad del defecto. No obstante, valores obtenidos para algunas relaciones geométricas arrojan valores elevados de la tensión local y poco sostenible estructuralmente en la practica, como por ejemplo Kt 17.08 con (R/H $0.05, \mathrm{H} / \mathrm{T} \quad 0.05$ y A/T 0.5$)$.

Mediante la base de datos de Kt obtenida a partir de los 75 modelos procesados con distintas relaciones adimensionales fue posible interpolar una función analítica. La diferencia maxima observada entre la función de Kt y los valores de la base de datos es menor al $5 \%$.

Mediante el uso de la expresión analítica obtenida se pueden diseñar ensayos de laboratorio para la determinacion de $\mathrm{Kt}$ en forma experimental adoptando relaciones geométricas que pertenezcan a distintas curvas de nivel. De este modo es posible racionalizar las determinaciones experimentales necesarias para un dado material.

\section{Agradecimientos}

Los autores desean agradecer el inestimable apoyo prestado por las siguientes instituciones: CONICET, CIC, UNMdP y Aluar S.A.

\section{References}

1. Radaj, D. Design and Analysis of Fatigue Resistant Welded Structures, Abington Publishing, Cambridge, England, 1990.

2. Sharp, M.L. Behavior and Design of Aluminum Structures, McGraw-Hill Inc, 1993.

3. Richards, K.G. Fatigue Strength of Welded Structures, The Welding Institute, Abington Hall, Cambridge, England, 1969.

4. Gurney, H. Fatigue of Welded Structures, Abington Publishing, Cambridge, England, 1990.

5. Nordmark, G.E.; Herbein, W.C.; Dickerson, P.B.; Montemarano, T.W. Effect of Weld Discontinuities on Fatigue of Aluminum Butt Joints, Welding Journal, 1987, v. $162-s / 173-s$.

6. Chapetti, M.D.; Otegui, J.L. A Technique to Produce Automatic Welds with Enhanced Fatigue Crack Propagation Lives Under Transverse Loading, Int.J.Pres.Ves. \& Piping, v. 70, p. 173-181, 1997.

7. Chapetti, M.D.; Otegui, J.L. Inportance of Toe Irregularity for Fatigue Resistance of Automatic Welds, Int.J Fatigue, v. 17, n. 8, p. 531-538, 1995.

8. Otegui, J.L.; Mohaupt, U.H.; Burns, D.J. Effect of Weld Process on Early Growth of Fatigue Cracks in Steel T Joints, Int.J Fatigue, v. 13, n. 1, p. 45-48, 1991.

9. Otegui, J.L.; Burns, D.J.; Kerr, H.W.; Mohaupt, U.H. Growth and Coalescence of Fatigue Cracks at Weld Toes in Steel, Int.J Pres.Ves. \& Piping, v. 48, p. 129-165, 1991.

10. Agerskov, H.; Petersen, R.I.; Martínez, L.L. An Investigation on Fatigue in High-Strength Steel Offshore Structures, IIW Doc. XIII, p. 1670, 1997.

11. Gosch, T.; Petershagen, H. Influence of Undercuts on the Fatigue Strength of Welded Joints, Schweissen \& Schneiden, 3/97, E 44/48.

12. ANSI/AWS D1.1 Structural Welding Code - Steels, American Welding Society, 1986.

13. Eurocode 3, Design of Steel Structures (Final Draft), Part 1 - General Rules and Rules for Buildings, Commission of European Communities, Brusels, 1988.

14. Offshore Installations: Guidance on Design and Construction, Department of Energy, (HMSO), London, 1984.

15. Fayard, J.L.; Bignonnet, A. Fatigue Assessment of Automobile Welded Structures, Doc. IIW-XIII, p. 1643, 1996.

16. Soya, I. Fatigue Strength Evaluation of Welded Joint Based on Local Approach and Structural Stress Concentration Factors at Weld Toe, Doc. IIW -XIII, p. 1672, 1997.

17. Janosch, J.J.; Debiez, S. Influence of the Shape of Undercut on the Fatigue Strength of Fillet Welded Assemblies - Application of the Local Approach, IIW Doc. XIII, p. 1694, 1997.

18. Langhaar, H.L. Dimensional Analysis and Theory of Models, J Wiley \& Son. 